\title{
Adaptive Dynamic Programming Boundary Control of Uncertain Coupled Semi-Linear Parabolic PDE
}

\author{
B. Talaei ${ }^{1}$, S. Jagannathan ${ }^{1}$, and J. Singler ${ }^{2}$
}

\begin{abstract}
This paper develops an adaptive dynamic programming (ADP) based near optimal boundary control of distributed parameter systems (DPS) governed by uncertain coupled semi-linear parabolic partial differential equations (PDE) under Neumann boundary control condition. First, Hamilton-Jacobi-Bellman (HJB) equation is formulated without any model reduction and the optimal control policy is derived. Subsequently, a novel identifier is developed to estimate the unknown nonlinearity in PDE dynamics. Accordingly, the sub-optimal control policy is obtained by forward-in-time estimation of the value functional using a neural network (NN) online approximator and the identifier. Adaptive tuning laws are proposed for learning the value functional online. Local ultimate boundedness (UB) of the closed-loop system is verified by using Lyapunov theory. The performance of proposed controller is verified via simulation on an unstable coupled diffusion reaction process.
\end{abstract}

\section{INTRODUCTION}

Significant number of industrial processes are inherently distributed in space so that their behavior depend on spatial position and time [1]-[2]. These systems are usually described by a set of partial differential equations (PDE) with homogenous or mixed boundary conditions. In particular, coupled semi-linear parabolic PDEs represent a wide range of industrial distributed parameter systems (DPS) such as reaction-diffusion processes [1] and fluid flow [2].

Control of DPS modeled as single PDE by using operator theory has been extensively studied in the literature [3]-[4] and also extended to boundary control [5] which is mathematically more involved and physically more practical and relevant. However, in many DPS processes, there are interactions between different components and therefore it is desirable to simultaneously control a set of variables with dynamics that are modeled by coupled PDE equations.

In [6], boundary control of coupled PDE using operator theory has been studied in detail with particular attention to parabolic-hyperbolic coupling arising in acoustics. After the development of backstepping as a new approach for boundary control of PDE in the last decade [7], control of higher dimensional coupled PDE dynamics using this method was also studied in recent years [8]-[10]. In [8], the

${ }^{1}$ Department of Electrical and Computer Engineering, ${ }^{2}$ Department of Mathematics and Statistics, Missouri University of Science and Technology, Rolla, MO, 65409. E-mail: behzad.talaei@mst.edu, sarangap@mst.edu, singlerj@mst.edu. Research supported in part by NSF grant ECCS\#1128281 and Intelligent Systems Center. linearized model of thermal-fluid convection has been treated by combining backstepping and Fourier series methods. In [9], a system of coupled first-order hyperbolic linear PDE with single boundary input was considered. In [10], the stabilization of $n$ linear diffusion reaction processes coupled with reaction processes was studied.

After the development of adaptive dynamic programming (ADP) [11] to solve optimal control problems in real-time for systems with large dimensional state spaces, ADP based control schemes were also developed for DPS However, since dealing with PDE dynamics and infinite dimensional state spaces were difficult, the conventional method extracts a finite dimensional space prior to ADP controller design [12]-[13], and the references therein.

This paper addresses a neural network $(\mathrm{NN})$ based near optimal boundary control scheme using ADP for uncertain DPS expressed as coupled semi-linear parabolic PDE without using any model reduction. In contrast to [12]-[13], the optimal control problem is formulated in the original PDE domain and solved forward-in-time without using any finite dimensional model approximation prior to the control design. Moreover, a NN identifier is proposed for the online estimation of the nonlinearity in the PDE so that the need for availability of system dynamics is relaxed.

After defining an appropriate cost functional, the HJB equation is derived in the infinite dimensional space and the optimal control policy is obtained based on necessary conditions of optimality. Subsequently, motivated by the fact that the nonlinearity in coupled PDE dynamics may be highly uncertain, a NN identifier is proposed to estimate it online. By approximating the optimal value functional based on a novel adaptive framework, the actual control policy is derived. Instead of using value or policy iterations [14] which have been considered unsuitable for real time control, the value functional weights are tuned online based on conventional adaptive techniques. Eventually, Lyapunov analysis is utilized to demonstrate the ultimate boundedness (UB) of the closed-loop system. Simulation results confirm the effectiveness of proposed control approach on an unstable coupled diffusion-reaction process.

Throughout the paper, |..| stands for Euclidean norm for a vector or Frobenius norm for a matrix. We recall the inequality \|\|$_{2} \leq\|\|_{F}$ with \|\|$_{2}$ being the induced 2 norm. We also define for $\forall x \in\left[\mathcal{L}_{2}(0, l)\right]^{n},\|x\|_{\mathcal{L}_{2}}=\left(\int_{0}^{l}\|x(z)\|_{2}^{2} d z\right)^{\frac{1}{2}}$. 


\section{ADAPTIVE OPTIMAL CONTROL}

The class of DPS considered in this paper is described by following semi-linear coupled parabolic PDE dynamics with Neumann boundary control at $z=l$ as

$$
\begin{array}{ll}
\frac{\partial x}{\partial t}=A \frac{\partial^{2} x}{\partial z^{2}}+f(x, z) & \\
\left.\frac{\partial x}{\partial z}\right|_{z=l}=G u(t), & z \in[0, l] \\
\left.\frac{\partial x}{\partial z}\right|_{z=0}=-P x(0, t), & x(z, 0)=x_{0}(z),
\end{array}
$$

where $x(z, t)=\left[x_{1}(z, t), \ldots, x_{n}(z, t)\right]^{T} \in X$ is the state vector, $X \in\left[\mathscr{H}_{2}(0, l)\right]^{n}$ being the solution space of PDE with $\mathscr{H}_{2}$ being the Sobolev space of second order, $t$ represents time, $z \in[0, l], l>0$ being the spatial variable, $A \in \mathbb{R}^{n \times n}$ is a constant diagonal matrix with $A_{i, i}=a_{i}>0$, $f(x, z)=\left[f_{1}(x, z), \ldots, f_{n}(x, z)\right]^{T} \in[C(0, l)]^{n}$ is an unknown Lipschitz continuous nonlinear vector function, and $\frac{\partial x}{\partial t}$ and $\frac{\partial^{2} x}{\partial z^{2}}$ denotes the time and second spatial derivatives of state $x$. Since $t \in[0, \infty)$ and $z \in[0, l]$, the PDE domain is given by $D \subset[0, \infty) \times[0, l]^{n}$.Moreover, $u(t)=\left[u_{1}(t), \ldots, u_{n}(t)\right]^{T} \in \mathbb{R}^{n}$ is the vector of boundary control input signals, $G, P \in \mathbb{R}^{n \times n}$ are known constant matrices and $x_{0}(z) \in C_{2}(0, l)$ represents the initial condition of the state with $C_{2}$ being the space of second order differentiable functions.

The goal is to provide a continuous control input that minimizes the cost functional over infinite horizon given by $\bar{V}\left(x, t_{0}, u\right)=\int_{t_{0}}^{\infty}\left(Q(x(z, t))+u^{T}(t) R u(t)\right) d t$,

where $R \in \mathbb{R}^{n \times n}$ is a positive definite matrix and $Q(x)$ is an integral functional with a nonlinear integrand function $q:\left[\mathscr{H}_{2}\right]^{n} \times[0, l] \rightarrow C_{2}[0, l]$ expressed as

$Q(x)=\int_{0}^{l} q(x(z, t), z) d z$.

It is assumed that $Q(x)$ is also positive definite i.e. $Q(0)=0$ and $Q(x) \geq q_{\min }\|x\|_{\mathcal{L}_{2}}^{2}$. In contrast to finite dimensional systems where the state is denoted by a vector, there are infinitely many states $x(z, t)$ in DPS that are continuous on the domain $[0, l]$. Therefore, integral functional such as (3) are necessary [4] for definition of $Q(x)$ instead of finite dimensional summations in conventional optimal control [11].
If the optimal cost functional is represented by $\bar{V}^{*}$, assume that it can also be represented by a state dependent nonlinear integral functional as

$$
\bar{V}^{*}(x, t)=\int_{0}^{l} V^{*}(x(z, t), z) d z,
$$

where $V^{*}(x(z, t), z):\left[\mathscr{H}_{2}\right]^{n} \times[0, l] \rightarrow C_{1}[0, l]$. Note that there is no restriction on the form of $V^{*}(.,$.$) . By taking the time$ interval $[t, t+\delta t), \bar{V}(x, t, u)$ in (2) can be expressed in the recursive form as

$\bar{V}(x, t, u)=\int_{t}^{t+\delta t}\left\{Q(x)+u^{T} R u\right\} d t+\bar{V}(x, t+\delta t, u)$.

Hence,

$\bar{V}^{*}(x, t)=\min _{u}\left\{\int_{t}^{t+\delta t}\left\{Q(x)+u^{T} R u\right\} d t+\bar{V}(x, t+\delta t, u)\right\}$.

Based on the principle of optimality, equation (6) becomes

$\bar{V}^{*}(x, t)=\min _{u}\left\{\int_{t}^{t+\delta t}\left\{Q(x)+u^{T} R u\right\} d t+\bar{V}^{*}(x, t+\delta t)\right\}$.

It is assumed that $\bar{V}^{*}(x, t)$ is Gâteaux analytic [15] in neighborhood of $(x(z, t), t)$, i.e. its directional derivative with respect to $x$ trajectory is available. If an integral functional $Y(x)=\int_{0}^{l} y(x) d z$ with $y(x)$ being a function of $x$ is Gâteaux analytic, according to calculus of variations [15], $Y(x+\delta x)$ can be represented by its first order approximation as

$$
Y(x+\delta x) \approx Y(x)+\int_{0}^{l} y_{x}^{T} \delta x d z
$$

where $y_{x}=\frac{\partial y}{\partial x}$ is the derivative of $y(x)$ with respect to $x$ and $\delta x$ represents an infinitesimal change in $x$. Therefore $\bar{V}^{*}(x, t+\delta t)$ can be represented in its first order approximation form as

$\bar{V}^{*}(x, t+\delta t) \approx \bar{V}^{*}(x, t)+\int_{0}^{l} V_{x}^{* T} \delta x d z+\frac{\partial \bar{V}^{*}}{\partial t} \delta t$,

where $V_{x}^{*}$ is the first order Gâteaux derivative of $V^{*}$ with respect to $x$ and $\delta x$ is an infinitesimal variation in state $x$ induced by $\delta t$ change in time. Since for infinite horizon optimal control, the cost functional $U^{*}$ does not explicitly depend on time, $\frac{\partial \bar{V}^{*}}{\partial t}=0$. Therefore, Substituting approximation (9) into (7), canceling $\bar{V}^{*}(x, t)$ on both sides, dividing through by $\delta t$, letting $\delta t \rightarrow 0$ and finally 
substituting PDE dynamics (1) for $\frac{\partial x}{\partial t}$ results in the Hamilton Jacobi Bellman (HJB) equation given by

$$
0=\min _{u}\left\{Q(x)+u^{T} R u+\int_{0}^{l} V_{x}^{* T}\left(A \frac{\partial^{2} x}{\partial z^{2}}+f(x, z)\right) d z\right\} .
$$

Subsequently, the Hamiltonian is defined as

$$
H=Q(x)+u^{T} R u+\int_{0}^{l} V_{x}^{* T}\left(A \frac{\partial^{2} x}{\partial z^{2}}+f(x, z)\right) d z .
$$

Using integration by parts, one can write Hamiltonian as $H=Q(x)+u^{T} R u+\int_{0}^{l} V_{x}^{* T} f(x, z) d z$

$-\int_{0}^{l} \frac{\partial V_{x}^{* T}}{\partial z} A \frac{\partial x}{\partial z} d z+V_{x}^{* T}(x, l) A G u+V_{x}^{* T}(x, 0) P A x(0)$.

The necessary condition for optimality implies that for the control policy to be optimal $\frac{d H}{d u}=0$. Therefore,

$2 R u+G^{T} A V_{x}^{*}(x, l)=0 \Rightarrow u^{*}=-\frac{1}{2} R^{-1} G^{T} A V_{x}^{*}(x, l)$.

Note that since $A$ is a diagonal matrix $A^{T}=A$. Taking $E^{T}=G^{T} A$ and substituting the optimal control (13) in (12), the HJB equation is given by

$$
\begin{aligned}
& H^{*}=0=Q(x)+\int_{0}^{l} V_{x}^{*} T f(x, z) d z-\int_{0}^{l} \frac{\partial V_{x}^{* T}}{\partial z} A \frac{\partial x}{\partial z} d z \\
& -\frac{1}{4} V_{x}^{* T}(l) E R^{-1} E^{T} V_{x}^{*}(l)+V_{x}^{* T}(0) P x(0),
\end{aligned}
$$

where $V_{x}^{*}(x, z)$ is represented by $V_{x}^{*}(z)$ for brevity.

Since the nonlinear function $f(x, z)$ in the system dynamics is unknown, an identifier is introduced prior to control synthesis. Therefore, in the next section the design of $\mathrm{NN}$ identifier will be explained.

\section{A. Identifier Design}

Since $f$ is a function over $X \times[0, l] \rightarrow[C(0, l)]^{n}$, there exists a compact set $\Omega \subset X \times[0, l]$, such that for $(x, z) \in \Omega$ by choosing a set of smooth bounded basis functions $\sigma_{f i}:\left[\mathscr{H}_{2}\right]^{n} \times[0, l] \rightarrow[C(0, l)]^{m}$, the function $f(x, z)$ can be written as

$$
f(x, z)=W_{f}^{T} \sigma_{f}(x, z)+\varepsilon_{f}(x, z),
$$

where $W_{f} \in \mathbb{R}^{m \times n}$ represents NN identifier target weight matrix with bound $\left\|W_{f}\right\| \leq W_{f M}$ and $\varepsilon_{f}(x, z)$ is the approximation error which is also assumed to be bounded above such that $\left\|\varepsilon_{f}(x, z)\right\| \leq \varepsilon_{f M}$ for all $x$ and $z$. The estimation error bound $\varepsilon_{f M}$ can be made arbitrarily small by increasing the number of basis functions.

Remark 1: Existence of compact set $\Omega$ can be deduced from the fact that as long as the domain $D$ of PDE (1) is a bounded set satisfying the cone condition, any $\mathcal{L}_{2}$ bounded and closed set in the solution space $X$ of PDE forms a compact set according to compact embedding theorem [16].

Naturally, the identifier dynamics can be provided by $\hat{f}(x, z)=\hat{W}_{f}^{T} \sigma_{f}(x, z)$.

In order to find the tuning law for $\hat{W}_{f}$, a state estimator with following PDE dynamics is initially considered as

$\frac{\partial \hat{x}}{\partial t}=A \frac{\partial^{2} \hat{x}}{\partial z^{2}}+\hat{f}(x, z)+K \tilde{x}$,

$\frac{\partial \hat{x}}{\partial z}(l)=G u, \quad \frac{\partial \hat{x}}{\partial z}(0)=-P x(0)$,

where $\tilde{x}=x-\hat{x}$ is the state estimation error and $K \in \mathbb{R}^{n \times n}$ is a positive definite matrix. Subsequently, the NN tuning law for $\hat{W}_{f}$ is provided by

$$
\dot{\hat{W}}_{f}=-\alpha_{f} \hat{W}_{f}+\int_{0}^{l} \sigma_{f}(x, z) \tilde{x}^{T} d z,
$$

where $\alpha_{f}>0$ is the design parameter. The second term in (18) integrates the state estimation error $\tilde{x}$ with different basis functions $\sigma_{f i}(1 \leq i \leq m)$ as integral kernels. Therefore, if estimation $\hat{f}$ and consequently $\hat{x}$ in (17) is unacceptable, the second term in (18) will adjust the weights associated with $\sigma_{f i}$ s close to their target value. Accordingly, the state estimation error $\tilde{x}$ dynamics will be governed by

$\frac{\partial \tilde{x}}{\partial t}=A \frac{\partial^{2} \tilde{x}}{\partial z^{2}}+\tilde{W}_{f} \sigma_{f}(x, z)+\varepsilon_{f}(x, z)-K \tilde{x}$,

$\left.\frac{\partial \tilde{x}}{\partial z}\right|_{z=l}=0,\left.\quad \frac{\partial \tilde{x}}{\partial z}\right|_{z=0}=0$,

where $\tilde{W}_{f}=W_{f}-\hat{W}_{f}$. Noting $\dot{\tilde{W}}_{f}=-\dot{\hat{W}}_{f}$, the dynamics of NN identifier weight estimation error can be represented as $\dot{\tilde{W}}_{f}=\alpha_{f} \hat{W}_{f}-\int \sigma_{f}(x, z) \tilde{x}^{T} d z$.

Since an estimate of system dynamics is now available, in the next section the ADP approximate optimal control will be addressed.

\section{B. Near Optimal Controller Design}

By a simple study of equations (13) and (14), it would be clear that in order to find the optimal control policy, $V_{x}^{*}(x, z)$ should be found by solving equation (14) and then substituted in the control input (13). Equation (14) is generally a nonlinear quadratic partial integro-differential equation (PIDE) and therefore it has no closed form solution. Consequently, the objective is to find a suitable structure for estimation of $V^{*}(x, z)$ in (14). For this purpose, since $V^{*}(x, z)$ is an integral functional over $\left[\mathscr{H}_{2}\right]^{n} \times[0, l] \rightarrow C_{2}[0, l]$, there exists a set of smooth 
bounded basis functions $\Phi_{i}:\left[\mathscr{H}_{2}\right]^{n} \times[0, l] \rightarrow C_{2}[0, l]$, such that

$V^{*}(x, z)=W_{\Phi}^{T} \Phi(x, z)+\varepsilon_{V}(x, z)$,

where $W_{\Phi} \in \mathbb{R}^{r}$ is the target $\mathrm{NN}$ weight vector with $\left\|W_{\Phi}\right\| \leq W_{\Phi M}$. Note that It is assumed that the uniform norm of derivative of approximation error with respect to $x$, i.e. $\left\|\varepsilon_{V x}\right\|$ and its second and third derivatives with respect to $z$, i.e. $\left\|\frac{\partial \varepsilon_{V x}}{\partial z}\right\|$ and $\left\|\frac{\partial^{2} \varepsilon_{V x}}{\partial z^{2}}\right\|$ are bounded by $\varepsilon_{V x M}, \varepsilon_{V x z M}$ and $\varepsilon_{V x z z M}$, respectively. The estimation error $\varepsilon_{V}$ can be made arbitrarily small by increasing the number of basis functions.

Therefore the optimal control input can be represented as

$u^{*}=-\frac{1}{2} R^{-1} E^{T} \Phi_{x}^{T}(x, l) W_{\Phi}+\varepsilon_{u}$,

where $\varepsilon_{u}=\frac{-1}{2} R^{-1} E^{T} \varepsilon_{V x}(x, l)$. Consequently, HJB equation can be represented in its approximated form as

$$
\begin{aligned}
& H^{*}=0=Q(x)+\int_{0}^{l} W_{\Phi}^{T} \Phi_{x}(z) W_{f}^{T} \sigma_{f}(z) d z-\int_{0}^{l} W_{\Phi}^{T} \frac{\partial \Phi_{x}}{\partial z} \\
& A \frac{\partial x}{\partial z} d z-\frac{1}{4} W_{\Phi}^{T} \Phi_{x}(l) E R^{-1} E^{T} \Phi_{x}^{T}(l) W_{\Phi} \\
& +W_{\Phi}^{T} \Phi_{x}(0) P A x(0)+\varepsilon_{H},
\end{aligned}
$$

where $\Phi(z)$ and $\sigma_{f}(z)$ are the abbreviation for $\Phi(x, z)$ and $\sigma_{f}(x, z)$, respectively and $\varepsilon_{H}$ can be derived as

$\varepsilon_{H}=\int_{0}^{l} \varepsilon_{V x}^{T}(z) f(x, z) d z+\int_{0}^{l} W_{\Phi} \Phi_{x}^{T}(z) \varepsilon_{f}(x, z) d z$

$-\int_{0}^{l} \frac{\partial \varepsilon_{V x}^{T}}{\partial z} A \frac{\partial x}{\partial z} d z-\frac{1}{4} \varepsilon_{V x}^{T}(l) E R^{-1} E^{T} \varepsilon_{V x}(l)$

$-\frac{1}{2} W_{\Phi}^{T} \Phi_{x}(l) E R^{-1} E^{T} \varepsilon_{V x}(l)+\varepsilon_{V x}^{T}(0) P A x(0)$.

If $V^{*}(x, z)$ is estimated by

$\hat{V}^{*}(x, z)=\hat{W}_{\Phi}^{T} \Phi(x, z)$,

the approximated HJB can be represented as

$$
\begin{aligned}
& \hat{H}=Q(x)+\int_{0}^{l} \hat{W}_{\Phi}^{T} \Phi_{x}(z) \hat{W}_{f}^{T} \sigma_{f}(z) d z-\int_{0}^{l} \hat{W}_{\Phi}^{T} \frac{\partial \Phi_{x}}{\partial z} A \frac{\partial x}{\partial z} \\
& d z-\frac{1}{4} \hat{W}_{\Phi}^{T} \Phi_{x}(l) E R^{-1} E^{T} \Phi_{x}{ }^{T}(l) \hat{W}_{\Phi}+\hat{W}_{\Phi}^{T} \Phi_{x}(0) P A x(0) .
\end{aligned}
$$

The control policy becomes

$$
\hat{u}=-\frac{1}{2} R^{-1} E^{T} \Phi_{x}^{T}(l) \hat{W}_{\Phi} .
$$

Select the value function NN weight tuning law as

$$
\dot{\hat{W}}_{\Phi}=-\alpha_{1} \frac{\omega_{N}}{\zeta_{N}^{2}} \hat{H}-\alpha_{2} \hat{W}_{\Phi}-\alpha_{3} \hat{W}_{\Phi}\left\|\hat{W}_{\Phi}\right\|^{2}
$$

where

$\omega_{N}=\int_{0}^{l} \Phi_{x}(z) \hat{W}_{f}^{T} \sigma_{f}(z) d z-\int_{0}^{l} \frac{\partial \Phi_{x}}{\partial z}(z) A \frac{\partial x}{\partial z} d z-\frac{1}{2} \Phi(l) E R^{-1}$

$E^{T} \Phi^{T}(l) \hat{W}_{\Phi}+\Phi_{x}(0) P A x(0), \zeta_{N}=c_{1}\|x\|_{\mathcal{L}_{2}}+c_{2}\|x(l)\|+c_{3}\|x(0)\|$

$+c_{4}$ and $\alpha_{1}, \alpha_{2}, \alpha_{3}, c_{1}, c_{2}, c_{3}, c_{4}$ are suitable positive design parameters. Here the first term in tuning law (28) reduces the approximated Hamiltonian while the other two terms are necessary for showing the stability of the system based on Lyapunov criteria that will be explained in the proof.

\section{STABILITY ANALYSIS}

Theorem 1 (Boundedness of $N N$ identifier): Let the initial NN identifier weight estimation error $\tilde{W}_{f}$ and state estimation error $\tilde{x}$ be residing in a compact set $\widetilde{J}_{1}$, and the proposed $\mathrm{NN}$ identifier, the state estimator and $\mathrm{NN}$ weight tuning law be provided by (16), (17) and (18), respectively. In the presence of bounded inputs, there exists a positive definite matrix $K$ with minimum eigenvalue $\lambda_{\text {min }}(K)>1 / 2$ and tuning parameter $\alpha_{f}>0$ for the identifier weight update law such that the state estimation error $\tilde{x}$ and weight estimation error $\tilde{W}_{f}$ are all $U U B$.

Proof: Omitted because of space constraints.

Lemma 1: The following inequality

$\left|\varepsilon_{H}\right| \leq c_{H 1}\|x\|_{\mathcal{L}_{2}}+c_{H 2}\|x(l)\|+c_{H 3}\|x(0)\|+c_{H 4}$

holds where $c_{H 1}, c_{H 2}$ and $c_{H 3}$ are positive constants. Moreover note that $\left|\varepsilon_{H}\right| \leq \xi_{N M} \zeta_{N}$ where $\zeta_{N}$ is defined in the update law (28) and $\xi_{N M}$ is a positive constant.

Proof: Refer to appendix.

Theorem 2 (Performance of the state feedback $N N$ controller): Consider the DPS given by (1) and let the NN identifier, the state estimator and NN weight update law be provided by (16), (17) and (18) respectively. Moreover, let control policy and tuning law for value function weights be provided by (27) and (28), respectively where $0<\alpha_{1}<1$, $\alpha_{2}>\alpha_{3}$ and $\alpha_{3}>\theta_{\alpha_{3}}^{N}$ with $\theta_{\alpha_{3}}^{N}$ being a positive constant. Consequently, the system state $x$, state estimation error $\tilde{x}$ and weight estimation errors $\tilde{W}_{f}$ and $\tilde{W}_{\Phi}$ are all UB.

Proof: Refer to appendix.

In the next section, the performance of proposed ADP based NN controller is examined by simulation on a semilinear diffusion reaction process.

\section{Simulations}

The controller developed in Section II was simulated in order to verify its performance. Consider the nonlinear coupled unstable reaction-diffusion system given by 


$$
\begin{aligned}
& \frac{\partial x_{1}}{\partial t}=\frac{\partial^{2} x_{1}}{\partial z^{2}}+\beta_{T_{1}} e^{-\gamma /\left(1+x_{1}\right)} x_{2}+\frac{\beta_{\alpha 1}}{1+\delta\left(z-z_{0}\right)^{2}} x_{2} \\
& \frac{\partial x_{2}}{\partial t}=\frac{\partial^{2} x_{2}}{\partial z^{2}}+\beta_{T_{2}} e^{-\gamma /\left(1+x_{1}\right)} x_{2}+\frac{\beta_{\alpha 2}}{1+\delta\left(z-z_{0}\right)^{2}} x_{1} \\
& \left.\frac{\partial x}{\partial z}\right|_{z=0}=0,\left.\frac{\partial x}{\partial z}\right|_{z=1}=u \quad x(z, 0)=\sin (\pi z),
\end{aligned}
$$

where $u=\left[u_{1}, u_{2}\right]^{T}$ is the control input that exists only at $z=1$. Comparing (30) to dynamics (1) it can be deduced that $A=G=I_{2 \times 2}$. The following values were given to process parameters: $\beta_{T_{1}}=15, \quad \beta_{T_{2}}=20, \quad \beta_{\alpha_{1}}=8, \quad \beta_{\alpha_{2}}=12$, $\gamma=5.0, \delta=4$ and $z_{0}=0.5$. It was verified that the open loop system was highly unstable with these values for system parameters.

The cost functional that should be minimized was chosen to be

$V(x)=\int_{0}^{\infty}\left\{\int_{0}^{1} x^{T} x d z+u^{2}\right\} d t$

The NN activation functions for identifying $f(x)$ were chosen as $\sigma_{k}(x)=\frac{1}{1+\lambda\left(z-z_{i}\right)^{2}} \varphi_{j}(x), 1 \leq k \leq 18$ with

$z_{i} \in\{0.2,0.5,0.8\}, 1 \leq i \leq 3, \lambda=4$

$\varphi_{j}(x)=\rho\left(\left\{1, x_{1}, x_{1}^{2}, x_{2}, x_{2}^{2}, x_{1} x_{2}\right\}\right), \quad 1 \leq j \leq 6, \quad$ and

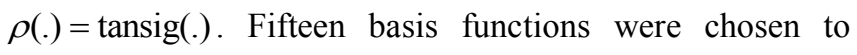
approximate $V^{*}$ with following structure

$\Phi_{k}(x, z)=\frac{0.5}{1+v\left(\bar{z}-\bar{z}_{i}\right)^{2}} \int_{\Delta_{i}} \psi_{j}(x) d z$

where $\quad 1 \leq k \leq 15, \quad \psi_{j}(x) \in \rho\left(\left\{x_{1}^{2}, x_{1}^{4}, x_{2}^{2}, x_{2}^{4}, x_{1}^{2} x_{2}^{2}\right\}\right)$, $1 \leq j \leq 5, z_{i} \in\{0.2,0.5,0.8\}$,

$\Delta_{i} \in\{(0,0.35),(0.35,0.65),(0.65,1)\}, 1 \leq i \leq 3$ and $v=8$.

The value functional update parameters were chosen to be $\alpha_{1}=0.5, \quad \alpha_{2}=0.2, \quad \alpha_{3}=0.15, \quad c_{4}=1, \quad c_{1}=0.2$, $c_{2}=c_{3}=0.02$ and identifier update parameters were selected as $\alpha_{f}=10$ and $K=100$. Note that design

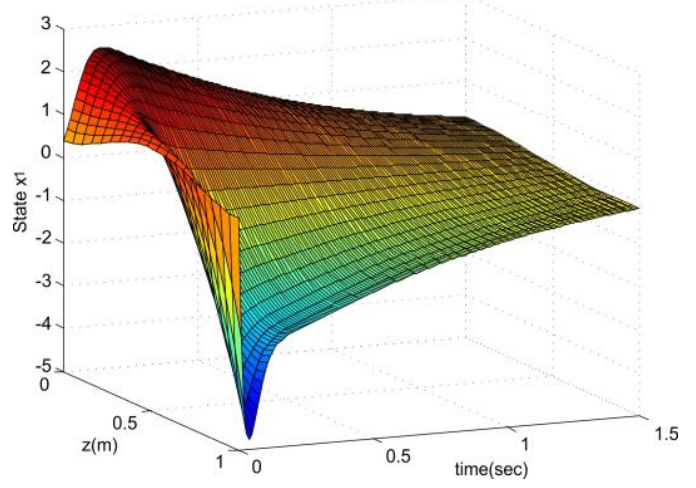

Fig 1 State history for state $x_{1}$.

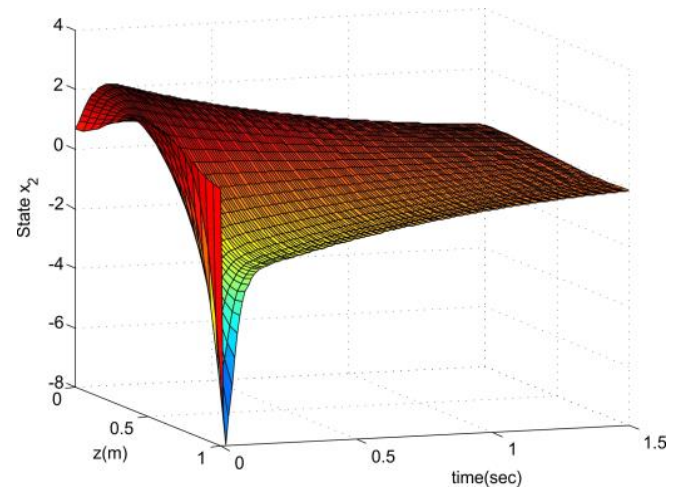

Fig 2 State history for state $x_{2}$.

conditions are satisfied with these parameters, i.e. $\alpha_{2}>\alpha_{3}$ and $\alpha_{3}>\theta_{\alpha_{3}}^{N} \simeq 0.1$. The controller was subsequently implemented on the system using MATLAB. The NN weights were updated and the controller was applied in realtime setting with step size of $1 \mathrm{msec}$ in order to have a convincing performance and computational rate.

As shown in Figs. 1 and 2, the proposed controller can stabilize the system to zero in less than 2 seconds. The control inputs which were the spatial derivatives of the states at the boundary $z=1$ are shown along with HJB error in Fig. 3. Since the system was unstable, its regulation required a considerable control effort at the beginning of simulation. Finally, faster convergence of HJB error to zero compared to control input verifies that the adaptive law (28) is capable of reducing the HJB error and achieving local optimality along system trajectories.

\section{CONCLUSIONS}

This paper developed a novel NN-based near optimal boundary control scheme for DPS governed by semi-linear parabolic PDE under Neumann boundary control condition without any model reduction prior to control design. By defining an integral cost functional and formulating the HJB equation based on calculus in infinite dimensional state space, a closed-form of optimal control policy was derived. The proposed adaptive NN framework made online approximation of optimal control policy along system
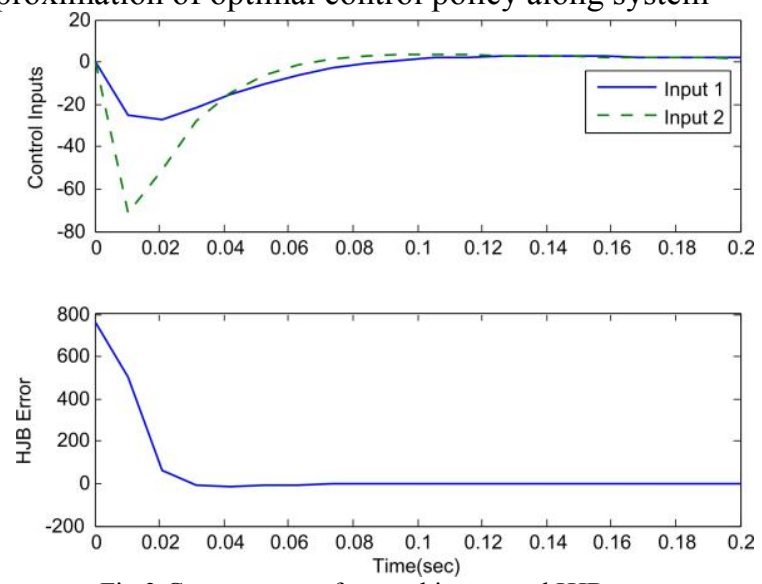

Fig 3 Convergence of control inputs and HJB error. 
trajectories possible whereas the PDE dynamics were partially unknown. Lyapunov stability analysis indicated that the error between approximated and truly optimal weights and state trajectories would remain UUB. The performance of controller in stabilizing and reducing the HJB error was successfully verified on a semi-linear diffusion process.

\section{APPENDIX}

Proof of Lemma 1: In order to avoid long derivations in the proof, we first define $x_{z}=\frac{\partial x}{\partial z}, x_{z z}=\frac{\partial^{2} x}{\partial z^{2}}, \Phi_{x}=\frac{\partial \Phi}{\partial x}$, $\Phi_{x z}=\frac{\partial \Phi_{x}}{\partial z}$ and $\varepsilon_{V x z}=\frac{\partial \varepsilon_{V x}}{\partial z}$. Using Cauchy's, Young's and Hölder's inequalities yield

$$
\begin{aligned}
& \left|\varepsilon_{H}\right| \leq l \varepsilon_{V x M} W_{f M} \sigma_{f M}+l \varepsilon_{V x M} \varepsilon_{f M}+l \varepsilon_{f M} W_{\Phi M} \Phi_{x M} \\
& +\sqrt{l} \varepsilon_{V x z z}\|A\|\|x\|_{\mathcal{L}_{2}}+\varepsilon_{V x z M}\|A\|\|x(l)\|+\varepsilon_{V x z M}\|A\| \\
& \|x(0)\|+\frac{1}{4} \delta \varepsilon_{V x M}^{2}+\frac{1}{2} \delta W_{\Phi M} \Phi_{x M} \varepsilon_{V x M}+\|P A\|\|x(0)\| \varepsilon_{V x M} \\
& =c_{H 1}\|x\|_{\mathcal{L}_{2}}+c_{H 2}\|x(l)\|+c_{H 3}\|x(0)\|+c_{H 4},
\end{aligned}
$$

where $\delta=\left\|E R^{-1} E^{T}\right\|, \quad c_{H 1}=\sqrt{l} \varepsilon_{V x z z M}\|A\|, c_{H 2}=\varepsilon_{V x z M}\|A\|$, $c_{H 3}=\varepsilon_{V x z M}\|A\|+\|P A\| \varepsilon_{V x M}$ and

$$
\begin{aligned}
& c_{H 4}=l \varepsilon_{V x M}\left(W_{f M} \sigma_{f M}+\varepsilon_{f M}\right)+l \varepsilon_{f M} W_{\Phi M} \Phi_{x M} \\
& +\frac{1}{4} \delta \varepsilon_{V x M}^{2}+\frac{1}{2} \delta W_{\Phi M} \Phi_{x M} \varepsilon_{V x M}
\end{aligned}
$$

Moreover, recalling $\zeta_{N}=c_{1}\|x\|_{\mathcal{L}_{2}}+c_{2}\|x(l)\|+c_{3}\|x(0)\|+c_{4}$, $\left|\varepsilon_{H}\right|$ can be expressed as

$$
\begin{aligned}
& \left|\varepsilon_{H}\right|=\left(\frac{c_{H 1}}{c_{1}}\right) c_{1}\|x\|_{\mathcal{L}_{2}}+\left(\frac{c_{H 2}}{c_{2}}\right) c_{2}\|x(l)\|+\left(\frac{c_{H 3}}{c_{3}}\right) c_{3}\|x(0)\| \\
& +\left(\frac{c_{H 4}}{c_{4}}\right) c_{4} \leq \xi_{N M} \zeta_{N}
\end{aligned}
$$

where $\xi_{N M}=\max \left\{\frac{c_{H 1}}{c_{1}}, \frac{c_{H 2}}{c_{2}}, \frac{c_{H 3}}{c_{3}}, \frac{c_{H 4}}{c_{4}}\right\}$.

Proof of Theorem 2: The details will be given later. Briefly, Consider the Lyapunov function

$$
\begin{aligned}
& L=\frac{1}{2} \tilde{W}_{\Phi}^{T} \tilde{W}_{\Phi}+\mu_{b} \int_{0}^{l} V^{*}(x) d z+\frac{1}{2} \mu_{c} \int_{0}^{l} \tilde{x}^{T} \tilde{x} d z \\
& +\frac{1}{2} \mu_{c} \operatorname{tr}\left(\tilde{W}_{f}^{T} \tilde{W}_{f}\right)
\end{aligned}
$$

where $\mu_{b}, \mu_{c}$ are positive constants. Taking its derivative $\dot{L}$ yields

$$
\begin{aligned}
& \dot{L} \leq-\mu_{b} q_{\text {min }}\|x\|_{\mathcal{L}_{2}}^{2}-\eta_{1}\left\|\tilde{W}_{\Phi}\right\|^{2}+\left[-\eta_{2}+\frac{1}{2} \mu_{b}\right]\left\|\tilde{W}_{\Phi}\right\|^{4} \\
& +\frac{3}{4} l^{2} W_{\Phi M}^{2} \Phi_{x M}^{2} \sigma_{f M}^{2}\left\|\tilde{W}_{f}\right\|^{2}-\mu_{c}\left(\lambda_{\min }(K)-\frac{1}{2}\right)\|\tilde{x}\|_{\mathcal{L}_{2}}^{2} \\
& -\frac{1}{2} \mu_{c} \alpha_{f}\left\|\tilde{W}_{f}\right\|^{2}+\varepsilon
\end{aligned}
$$

where $\eta_{1}, \eta_{2}$ and $\varepsilon$ are positive constants. Therefore, choosing $\mu_{b}<2 \eta_{2}$ and $\mu_{c}>\frac{3}{\alpha_{f}} l^{2} W_{\Phi M}^{2} \Phi_{x M}^{2} \sigma_{f M}^{2}, \quad \dot{L} \quad$ is always less than zero if $\|x\|_{\mathcal{L}_{2}}>\sqrt{\frac{\varepsilon}{\mu_{b} q_{\text {min }}}}$ or $\mid \tilde{W}_{\Phi} \|>\sqrt{\frac{\varepsilon}{\eta_{1}}}$ or $\|\tilde{x}\|_{\mathcal{L}_{2}}>\sqrt{\frac{\varepsilon}{\mu_{c}\left(\lambda_{\min }(K)-\frac{1}{2}\right)}} \quad$ or $\quad \mid \tilde{W}_{f} \|>\sqrt{\frac{4 \varepsilon}{\mu_{c} \alpha_{f}}}$.

Consequently, the closed-loop system is UUB.

Note that the bounds for $\|x\|_{\mathcal{L}_{2}}, \mid \tilde{W}_{\Phi}\|,\| \tilde{x} \|_{\mathcal{L}_{2}}$ and ॥ $\tilde{W}_{f} \|$, respectively can be made smaller by increasing the design parameters $q_{\min }, \alpha_{2}$ in $\eta_{1}=\frac{1}{2}\left(\alpha_{2}-\alpha_{3}\right), \lambda_{\text {min }}(K)$ and $\alpha_{f}$ in (20).

\section{REFERENCES}

[1] C. V. Pao, "Nonlinear parabolic and elliptic equations," Springer Science \& Business Media, 1992.

[2] Y. Giga, "Solutions for semilinear parabolic equations in $L_{p}$ and regularity of weak solutions of the Navier-Stokes system," Journal of differential equations, vol. 62, no. 2, 1986.

[3] M. Tucsnak and G. Weiss, "Observation and control for operator semigroups,” Springer Science \& Business Media, 2009.

[4] R. F. Curtain and H. Zwart, "An introduction to infinite-dimensional linear systems theory," vol. 21, Springer, 1995.

[5] I. Lasiecka and R. Triggiani, "Control Theory for Partial Differential Equations, Abstract Parabolic Systems: Continuous and Approximation Theories," Cambridge University Press, 2000.

[6] I. Lasiecka, "Mathematical Control of Coupled PDEs," SIAM, Vol. 75, 2002.

[7] M. Krstic and A. Smyshlyaev, "Boundary control of PDEs: A course on backstepping designs," SIAM, vol. 16, 2008.

[8] R. Vazquez, and M. Krstic, "Boundary Observer for OutputFeedback Stabilization of Thermal-Fluid Convection Loop," IEEE Transactions on Control Systems Technology, , vol.18, no.4, pp. 789,797 , July 2010

[9] F. Di Meglio, R. Vazquez, and M. Krstic, "Stabilization of a System of $\mathrm{n}+1$ Coupled First-Order Hyperbolic Linear PDEs With a Single Boundary Input," IEEE Transactions on Automatic Control, vol.58, no.12, pp. 3097-3111, Dec. 2013.

[10] A. Baccoli, Y. Orlov, and A. Pisano, "On the boundary control of coupled reaction-diffusion equations having the same diffusivity parameters," IEEE Conference on Decision and Control , 2014.

[11] F. L. Lewis and D. Liu, editors, "Reinforcement Learning and Approximate Dynamic Programming for Feedback Control," John Wiley/IEEE Press, Computational Intelligence Series, 2012.

[12] B. Talaei, H. Xu, and S. Jagannathan, "Near Optimal Boundary Control of Distributed Parameter Systems Modeled as Parabolic PDEs by using Finite Difference Neural Network Approximation," IEEE Conference on Decision and Control, 2014.

[13] B. Talaei, H. Xu, and S. Jagannathan, "Neural Network-based Near Optimal Constrained Control of Distributed Parameter Systems with Application to Diffusion-Reaction Processes," IEEE MSC, 2014.

[14] F. L. Lewis and D. Vrabie, "Reinforcement learning and adaptive dynamic programming for feedback control," IEEE Circuits and Systems Magazine, vol. 9, no. 3, pp. 32-50, 2009.

[15] D. R. Smith, "Variational methods in optimization," Courier Dover Publications, 1998.

[16] R. A. Adams and J. JF Fournier, "Sobolev spaces”, 2003. 